\title{
Search for long-lived neutral particles decaying into lepton-jets with the ATLAS Detector
}

\author{
Miriam Deborah Joy Diamond ${ }^{* \dagger}$ \\ University of Toronto \\ E-mail: mdiamondephysics.utoronto.ca
}

Several models of elementary particle physics beyond the Standard Model predict the existence of neutral particles that can be long lived and decay in collimated jets of light leptons and hadrons (lepton-jets). The present contribution describes the search for lepton-jets in $20.3 \mathrm{fb}^{-1}$ of $\sqrt{s}=8 \mathrm{TeV}$ proton-proton collision data recorded at the ATLAS detector in 2012, and compares the selected events with the Standard Model expectations and with various BSM predictions. Prospects for searches with $\sqrt{s}=13 \mathrm{TeV}$ ATLAS data are addressed.

Fourth Annual Large Hadron Collider Physics

13-18 June 2016

Lund, Sweden

${ }^{*}$ Speaker.

${ }^{\dagger}$ On behalf of the ATLAS Collaboration 


\section{Motivation and Benchmark Models}

A wide variety of Beyond the Standard Model (BSM) theories predict a hidden sector, weakly coupled to the visible sector, where the lightest hidden states are in the $\mathrm{MeV}$ to $\mathrm{GeV}$ mass range and decay back to Standard Model particles with significant branching fractions [1]. If produced at the LHC, these hidden-sector particles would be highly boosted, yielding highly-collimated decay products in the final state. Non-negligible lifetimes of the hidden-sector particles would result in decay vertices displaced relative to the primary vertex of the event. This motivates the search for "displaced lepton-jets" (dLJs): collimated jet-like structures, containing pair(s) of muons, electrons, and/or light hadrons, produced far from the primary vertex.

The dLJs are a distinctive expected signature of the dark photon $\left(A^{\prime}\right.$ or $\left.\gamma_{\mathrm{d}}\right)$, the heavy gauge boson of a BSM $U(1)$. In "vector portal" models, the dark photon can kinetically mix with the SM photon, due to the presence of a term $\frac{\varepsilon}{2} F^{\mu v} A_{\mu v}$ in the Langrangian. A smaller mixing parameter $\varepsilon$ yields a longer $\gamma_{d}$ lifetime. The branching fractions of the $\gamma_{d}$ depend on its mass, as documented in [2]. Our dLJ searches probe the regions of parameter space featuring long-lived, boosted low-mass $\gamma_{\mathrm{d}} \rightarrow l^{+} l^{-}$, where $l=e, \mu, \pi$. The pion is included here because the $\gamma_{\mathrm{d}}$ effectively couples to EM charge, and so its coupling to $\pi$ has the same strength as to $e$ and to $\mu$.

As a benchmark, we take the Falkowski-Ruderman-Volansky-Zupan (FRVZ) model introduced in [2]. As shown in Figure 1a, a Higgs boson (which may be SM-like or of a BSM heavy neutral variety) decays to heavy hidden fermions $f_{\mathrm{d} 2}$, each $f_{\mathrm{d} 2}$ decays to a $\gamma_{\mathrm{d}}$ and a Hidden Lightest Stable Particle (HLSP), and each $\gamma_{\mathrm{d}}$ decays to a dLJ. The opening angle of the constituents of a dLJ from such an event is typically small (Figure 1b).

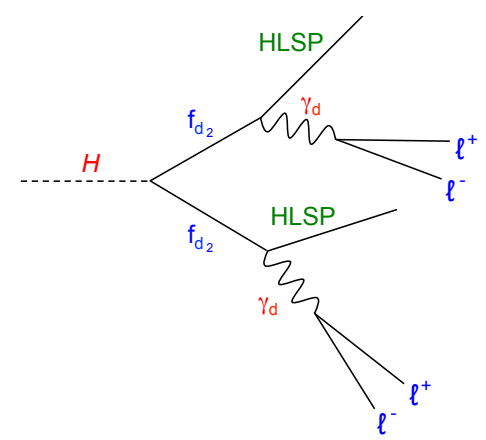

(a) The FRVZ benchmark model [2]

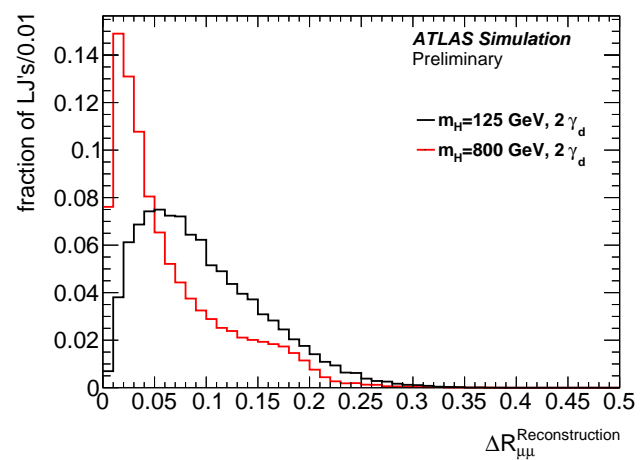

(b) Opening angle of constituents of reconstructed dLJs from $\gamma_{\mathrm{d}} \rightarrow \mu^{+} \mu^{-}$, evaluated in Monte Carlo [5].

Figure 1

\section{Search Strategy}

Our dLJ search targets $\gamma_{\mathrm{d}}$ decays that occur outside the ATLAS Inner Detector (ID), up to the Muon Spectrometer (MS) [6]. Muon pairs in dLJs appear as MS tracks with no associated ID tracks. Electron and pion pairs appear as "LJCalTracks", narrow isolated jets with low "EM 
fraction" (i.e. deposit much less energy in the EM Calorimeter than the Hadronic Calorimeter.) We categorize dLJs into Type-0, Type-1, and Type-2, as shown in Figure 2.
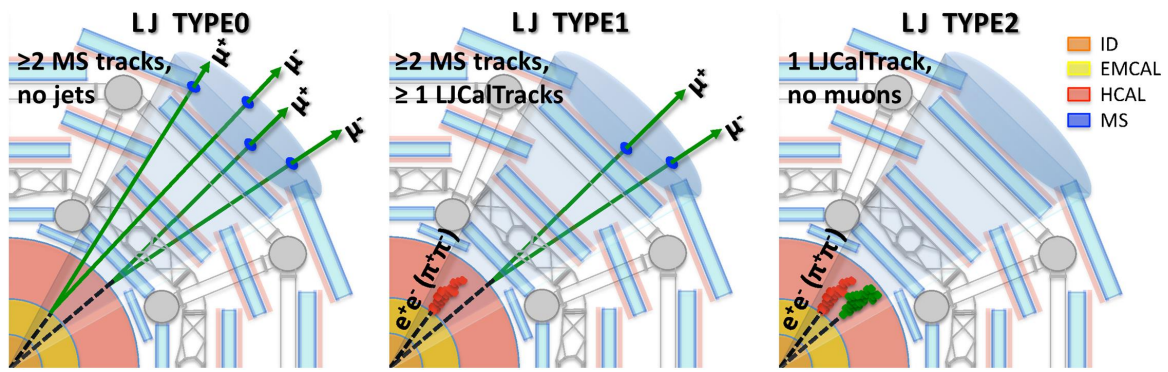

Figure 2: Categorization of dLJs based on particle species content [3].

Our dLJ-finding technique uses a clustering algorithm with $\Delta R=0.5$ cone, and special reconstruction considerations. These considerations are necessary because collimated final-state particles are difficult to reconstruct due to detector granularity, and the reconstruction of tracks from displaced vertices (a particularly difficult task outside the ID) requires the removal of primary vertex constraints that are used in standard ATLAS algorithms. The dLJ reconstruction efficiency [5] varies as a function of the transverse decay length of the $\gamma_{\mathrm{d}}$, as shown in Figure 3.
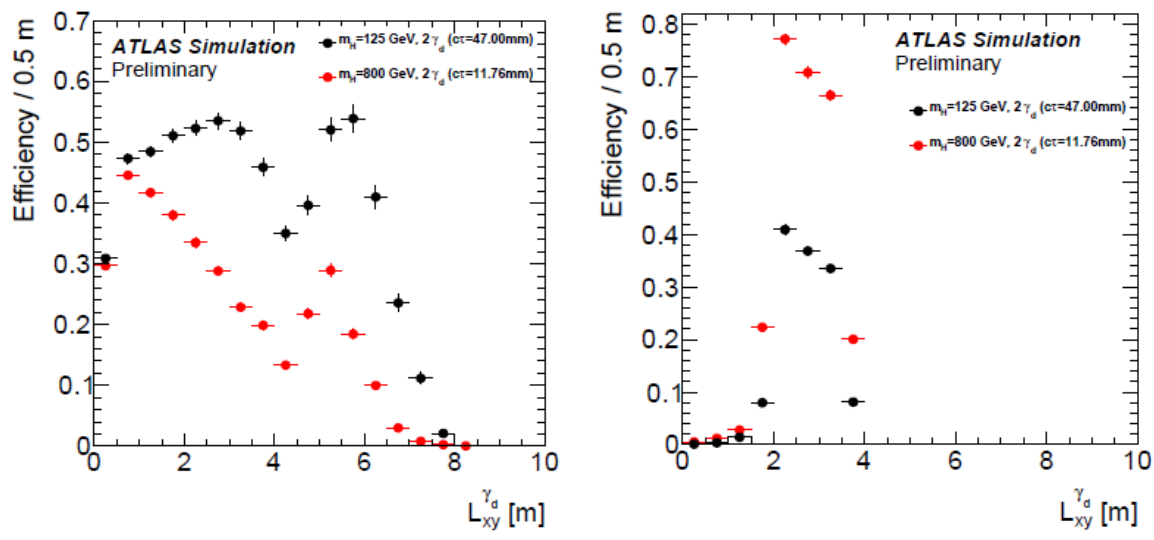

Figure 3: Reconstruction efficiency for dLJs, Type-0 (left) and Type-2 (right). The shapes of the curves are explained in [5]. Efficiency for Type- 1 is the product of efficiencies for Type- 0 and Type-2. Evaluated in Monte Carlo of the benchmark model for $2015(\sqrt{s}=13 \mathrm{TeV})$ conditions.

QCD multi-jets are the main background in the Type-2 and Type-1 dLJ search, and cosmicray muon bundles in the Type- 0 . Cosmic-ray muon energy deposits in the calorimeters, misreconstructed as LJCalTracks, are a secondary background in the Type-1 and Type-2. Beaminduced background (BIB), consisting of high-energy muons longitudinally crossing the detector with bremsstrahlung in the Hadronic Calorimeter, can also be mis-reconstructed as LJCalTracks.

\section{Triggers}

The ATLAS High-Level Trigger (HLT) system provide inline full reconstruction of the data in real time. The HLT triggers employed in the dLJ search are as follows: 
- Tri-muon: $3 \mathrm{MS}$ tracks $\left(p_{\mathrm{T}}>6 \mathrm{GeV}\right)$, no corresponding ID tracks

- Narrow-Scan: $2 \mathrm{MS}$ tracks (leading $p_{\mathrm{T}}>20 \mathrm{GeV}$, sub-leading $>6 \mathrm{GeV}$ ) in $\Delta R=0.5$ cone, no corresponding ID tracks

- CaloRatio: Jet $\left(p_{\mathrm{T}}>30 \mathrm{GeV}\right)$ with low EM fraction

The efficiency of each of these triggers [5], for the benchmark process, is shown in Table 1. The Narrow-Scan trigger, which provides a large increase in trigger efficiency, was newly introduced for 2015 data-taking.

\begin{tabular}{|c|c|c|c|}
\hline Trigger & $m_{H}=125 \mathrm{GeV}$, Run 1 & $m_{H}=125 \mathrm{GeV}$, Run 2 & $m_{H}=800 \mathrm{GeV}$, Run 2 \\
\hline \hline Tri-muon & $2.0 \%$ & $2.9 \%$ & $2.4 \%$ \\
\hline Narrow-Scan & $10.6 \%$ & - & $23.0 \%$ \\
\hline CaloRatio & $0.3 \%$ & $2.3 \%$ & $9.7 \%$ \\
\hline OR of all & $11.9 \%$ & $4.6 \%$ & $32.0 \%$ \\
\hline
\end{tabular}

Table 1: Efficiency of HLT triggers [5], evaluated in Monte Carlo of the benchmark model. Run 2 refers to 2015 data-taking conditions at $\sqrt{s}=13 \mathrm{TeV}$, and Run 1 to 2012 at $\sqrt{s}=8 \mathrm{TeV}$.

\section{Selection Requirements}

The dLJ candidate selection employs a set of cuts defined to optimize the signal significance:

- Jet Width, Jet EM Fraction, Jet Vertex Tagger: Rejects QCD jets, based on jet shape, energy deposition pattern, and incompatibility with the event's primary vertex (for Type-2 dLJs)

- Jet timing: Rejects mis-reconstructed cosmics (for Type-1, Type-2)

- Beam-Induced Background tagging: Rejects fake BIB jets accompanied by muon segments parallel to the beampipe (for Type-2)

Event selection, which requires a pair of dLJs in the event, follows. Cuts on two variables are employed, where the cut values are defined using a data-driven method to reduce QCD multi-jet contamination. This simplified matrix method, a simultaneous counting experiment in control and signal regions, assumes the background is factorizable in the two variables. The first is $\Sigma p_{\mathrm{T}}$, the scalar sum of the $p_{\mathrm{T}}$ of ID tracks in a $\Delta R=0.5$ cone around the dLJ. The second is $|\Delta \phi|$, the angular separation between the two dLJs. Events in the benchmark process generally have high $|\Delta \phi|$ and low $\Sigma p_{\mathrm{T}}$, while backgrounds are more prevalent elsewhere in the $\left(|\Delta \phi|, \Sigma p_{\mathrm{T}}\right)$ plane.

\section{Results}

A search for dLJ pairs was performed using the full 2012 ATLAS dataset at $\sqrt{s}=8 \mathrm{TeV}$ $\left(20.3 \mathrm{fb}^{-1}\right)$ [3]. To facilitate re-casting of the results, trigger and reconstruction efficiency tables (available as auxiliary material accompanying [3]) were produced as a function of the $c \tau$ and $p_{\mathrm{T}}$ of the $\gamma_{\mathrm{d}}$ using a dedicated Monte Carlo tool. Exclusion contours were established in the plane of 
$\varepsilon$ vs $\gamma_{\mathrm{d}}$ mass, in the context of vector portal models. These contours cover an area of parameter space untouched by other experiments (Figure 4a) - although the ATLAS limits depend upon an additional parameter, $\mathrm{BR}\left(H \rightarrow \gamma_{\mathrm{d}}+X\right)$. In the benchmark model, limits were set on $\sigma \times \mathrm{BR}(H \rightarrow$ $\left.\gamma_{\mathrm{d}}+X\right)$, thereby setting BR-dependent exclusion bounds on $c \tau\left(\gamma_{\mathrm{d}}\right)$ as (Figure $\left.4 \mathrm{~b}\right)$.

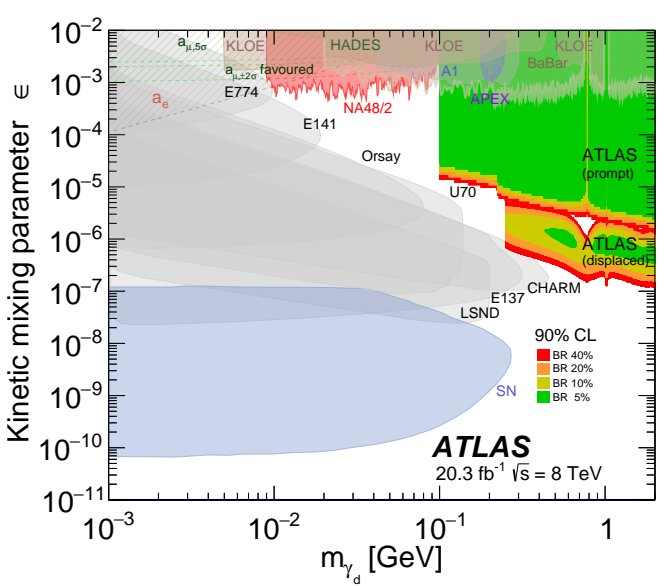

(a) Exclusion contours in the $\left(\varepsilon, m_{\gamma_{\mathrm{d}}}\right)$ plane. Contours labelled "prompt" are from a similar, complementary search for $\mathrm{LJ}$ pairs from dark photons with small or zero $c \tau$ [7].

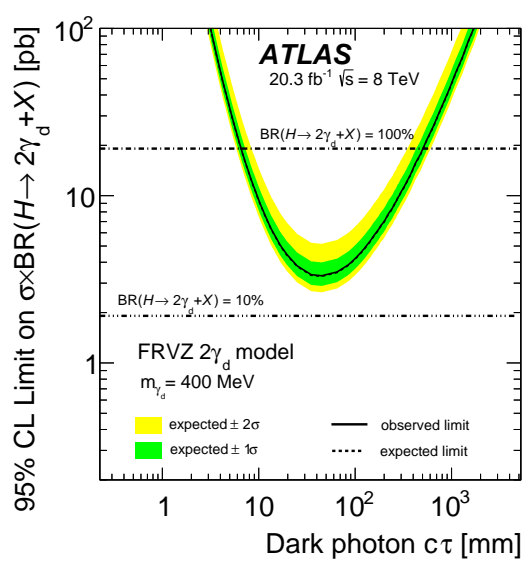

(b) Exclusion bounds on $c \tau\left(\gamma_{\mathrm{d}}\right)$ in the benchmark model, assuming SM Higgs produced via gluongluon fusion [3].

Figure 4

With $\sqrt{s}=13 \mathrm{TeV}$ 2015-2016 ATLAS data, higher Higgs production cross-sections [4] and increased trigger efficiency will greatly increase $\mathrm{dLJ}$ search sensitivity. It will be possible to extend the analysis to additional topologies: for example, processes with only one $\mathrm{dLJ}$ in the final state. Extensions to higher $\gamma_{\mathrm{d}}$ masses, higher Higgs masses (for BSM extended Higgs sectors), and associated Higgs production will also be possible.

\section{References}

[1] M. J. Strassler and K. M. Zurek, Phys. Lett. B 651 (2007) 374 doi:10.1016/j.physletb.2007.06.055 [hep-ph/0604261].

[2] A. Falkowski, J. T. Ruderman, T. Volansky and J. Zupan, JHEP 1005, 077 (2010) doi:10.1007/JHEP05(2010)077 [hep-ph/1002.2952].

[3] ATLAS Collaboration, JHEP 1411, 088 (2014) doi:10.1007/JHEP11(2014)088 [hep-ex/1409.0746].

[4] LHC Higgs Cross Section Working Group, S. Heinemeyer, C. Mariotti, G. Passarino, and R. Tanaka (Eds.), Handbook of LHC Higgs Cross Sections: 3. Higgs Properties, CERN-2013-004 (CERN, Geneva, 2013) [hep-ph/1307.1347].

[5] ATLAS Collaboration, ATL-PHYS-PUB-2016-010 (March 2016).

[6] ATLAS Collaboration, JINST 3 (2008) S08003 doi:10.1088/1748-0221/3/08/S08003

[7] ATLAS Collaboration, JHEP 1602, 062 (2016) doi:10.1007/JHEP02(2016)062 [hep-ex/1511.05542]. 